\title{
A Cross-Sectional Survey on Attitudes of Men Who Have Sex With Men Towards Anal Self-Examination for Detection of Anal Syphilis
}

Ei T Aung ( $\square$ eaung@mshc.org.au )

Melbourne Sexual Health Centre

Christopher K Fairley

Melbourne Sexual Health Centre

Jason J Ong

Melbourne Sexual Health Centre

Tiffany P Phillips

Melbourne Sexual Health Centre

Marcus Y Chen

Melbourne Sexual Health Centre

Julien Tran

Melbourne Sexual Health Centre

Kate Maddaford

Melbourne Sexual Health Centre

Elena R Rodriguez

Melbourne Sexual Health Centre

Eric P.F. Chow

Melbourne Sexual Health Centre

\section{Research Article}

Keywords: Survey, Sex, Anal Self-examination, Syphilis

Posted Date: July 26th, 2021

DOI: https://doi.org/10.21203/rs.3.rs-625835/v1

License: (c) (i) This work is licensed under a Creative Commons Attribution 4.0 International License.

Read Full License 


\section{Abstract}

Studies suggest men who have sex with men (MSM) practising receptive anal sex are more likely to present with secondary syphilis, implying primary anorectal lesions are likely to be missed. If men could detect anorectal lesions in the primary stage by regular anal self-examination (ASE), transmission could be reduced by early diagnosis and treatment. We aimed to explore the attitudes of MSM on performing ASE to detect primary anorectal syphilis. An online anonymous cross-sectional survey among MSM over 18 years of age living in Australia, was conducted between July and November 2020 and recruitment was from a sexual health clinic and social media. A total of 574 MSM completed the survey (median age: 34 [IQR 27-45]): 32\% (183) had previously performed ASE. Among 56\% (374) who had never performed ASE, $68 \%$ (250) would consider performing ASE in the future with a preferred median frequency of 2 times per 4 weeks (IQR: 1-4), whilst men who were already performing ASE were performing it at median 1 per 4 weeks (IQR: 0.2-3). Almost two-thirds of MSM who had never performed ASE were willing to adopt ASE practice in the future. Studies are required to determine the effectiveness of ASE for syphilis detection.

\section{Introduction}

Syphilis continues to rise among gay, bisexual and other men who have sex with men (MSM) in highcome countries despite numerous public health interventions to improve syphilis prevention and control ${ }^{1-5}$. Previous public health strategies included regular serological screenings of MSM who are considered at a higher risk of acquiring syphilis, improved contact tracing, and behavioural interventions to increase condom use ${ }^{6-8}$. Despite these measures, syphilis cases continue to rise prompting the need for additional strategies for syphilis control $^{9}$.

Reducing the duration of the infectious period is one of the main strategies underpinning interventions for syphilis control. Primary syphilis classically presents as a painless chancre at the point of inoculation ${ }^{10}$. These primary lesions may go unnoticed if they occur at hidden sites such as the vaginal or inside the anal canal. Cornelisse et al. reported that MSM who practised receptive penile-anal sex were four times more likely to present with secondary syphilis than those who practised insertive penile-anal sex, suggesting primary anorectal lesions are often missed ${ }^{11}$. Similarly, Gunn et al. reported that MSM who practised receptive penile-anal intercourse were $48 \%$ less likely to be diagnosed in the primary stage than other MSM, implying occult primary lesions are probably undiagnosed ${ }^{12}$. Furthermore, Peel et al. has found that routine scheduled 3-monthly syphilis screening during PrEP visits failed to detect a substantial proportion of infectious syphilis (58\% of primary syphilis and $44 \%$ of secondary syphilis), indicating that a 3-monthly serological screening alone may be insufficient for syphilis control among this population ${ }^{13}$. Recent studies have shown MSM with secondary syphilis often have T. pallidum detection at multiple sites, including the mouth and anus, suggesting that preventing the secondary stage of syphilis may also reduce the infectiousness ${ }^{14}$. It is hypothesised that if men examine their anus weekly in addition to 3 monthly routine screening, they might be able to detect primary anorectal lesions themselves and present for treatment before progressing to secondary syphilis, thereby reducing infectiousness. 
Anal self-examination (ASE) is a new concept for syphilis detection; however this practice has been introduced among MSM aged over 50 who are living with HIV to detect early anal cancer with good

acceptability ${ }^{15-20}$. A qualitative study among $20 \mathrm{MSM}$ recruited from a sexual health centre exploring their attitudes towards ASE for anal syphilis, and found that performing ASE was highly acceptable ${ }^{21}$. We designed this online anonymous survey study to further explore the perspectives of MSM recruited both at a sexual health centre and from the general community on ASE practice. This study aimed to investigate the attitudes and perceptions on ASE among MSM and their willingness to adopt the practice routinely for syphilis detection.

\section{Results}

Between July and November 2020, a total of 3154 SMS was sent to men attending MSHC. Of those, 620 (20\%) clicked the survey link and started the survey. Additionally, 89 surveys were received through social media from the community. Hence, a total of 709 responses were received. We excluded 141 participants, including 77 who did not complete surveys; 33 did not consent to participate the survey; 19 living outside Australia or declined to disclose where they lived; eight reported no sexual contact with another man in the last 12 months; and four self-identified as cis-female or female gender.

The remaining 565 men were included in the final analysis and the median age was 34 (IQR: 27-45) (Table 1). The majority were cis male $(98 \%, n=556)$. Most men reported sex with men only $(91 \%, n=514)$, and a small proportion reported having sex with both men and women $(9 \%, n=50)$. There were a few men who preferred not to report their sexual orientation $(1 \%, n=7)$. About one-third of men reported they had ever performed ASE $(32 \%, n=183)$ among 557 men who answered the question on previous experience of ASE. Table 1 shows the demographic characteristics of men who had performed ASE previously and who had never performed ASE with no statistical significance between the two groups. 
Table 1

Demographics of survey participants

\section{Ever performed ASE ( $\mathrm{N}=\quad$ Never performed ASE $(\mathrm{N}=$ \\ 183) 374)}

Age, median (Interquartile range) 35 (IQR: 29-47)

33 (IQR: 27-43)

0.041

\section{Gender $^{1}$}

Male

Non-binary/gender fluid

Other gender

\section{Education}

No post-school qualification ${ }^{\#}$

Certificate \& diploma* level

University degree ${ }^{\wedge}$

Sexual orientation

Gay

Bisexual

Prefer not to report

Anal sex position

Receptive anal sex

Versatile anal sex

Insertive anal sex

No anal or penetrative sex

HIV status and PrEP use
178 (97\%)

$4(2 \%)$

$1(0.6 \%)$

$4(1 \%)$

$5(1 \%)$
$365(98 \%)$

0.126

$51(13.3 \%)$

$76(20 \%)$

$247(66 \%)$

0.458

$334(89 \%)$

$35(9 \%)$

$5(1 \%)$

0.188

105 (28\%)

193 (52\%)

$69(18 \%)$

7 (2\%)

$3(2 \%)$

0.773

${ }^{1}$ Female was not reported in the table as those identified as female at birth were excluded.

* Certificate* I, II, III, IV level, advanced diploma and diploma level

^ Bachelor's degree, master's degree/graduate diploma/graduate certificate, postgraduate degree

\# No schooling completed, some secondary education - Years 9 and below, secondary education completed

Kruskal-Walis $\mathrm{H}$ test was used to compare the statistical significance of age between men who had previously performed ASE and men who had never performed ASE. Pearson's chi test was used to compare the other demographic characteristic between the two groups. 


\begin{tabular}{|c|c|c|c|}
\hline & $\begin{array}{l}\text { Ever performed ASE }(\mathrm{N}= \\
\text { 183) }\end{array}$ & $\begin{array}{l}\text { Never performed ASE }(\mathrm{N}= \\
\text { 374) }\end{array}$ & $\begin{array}{l}\text { p- } \\
\text { value }\end{array}$ \\
\hline Living with HIV & $46(25 \%)$ & $86(23 \%)$ & \\
\hline Taking PrEP & $71(39 \%)$ & $133(36 \%)$ & \\
\hline $\begin{array}{l}\text { Not living with HIV and not taking } \\
\text { PrEP }\end{array}$ & $61(33 \%)$ & $140(37 \%)$ & \\
\hline Unknown or prefer not to say & $5(3 \%)$ & $15(4 \%)$ & \\
\hline Previous syphilis infection & $72(39 \%)$ & $126(34 \%)$ & 0.346 \\
\hline \multicolumn{4}{|c|}{${ }^{1}$ Female was not reported in the table as those identified as female at birth were excluded. } \\
\hline \multicolumn{4}{|c|}{ * Certificate* I, II, III, IV level, advanced diploma and diploma level } \\
\hline \multicolumn{4}{|c|}{ ^ Bachelor's degree, master's degree/graduate diploma/graduate certificate, postgraduate degree } \\
\hline \multicolumn{4}{|c|}{$\begin{array}{l}\text { \# No schooling completed, some secondary education - Years } 9 \text { and below, secondary education } \\
\text { completed }\end{array}$} \\
\hline \multicolumn{4}{|c|}{$\begin{array}{l}\text { Kruskal-Walis } \mathrm{H} \text { test was used to compare the statistical significance of age between men who had } \\
\text { previously performed ASE and men who had never performed ASE. Pearson's chi test was used to } \\
\text { compare the other demographic characteristic between the two groups. }\end{array}$} \\
\hline
\end{tabular}

\section{Preferences for performing self-examination among men who had ever performed ASE}

The median frequency of performing ASE was one (IQR: 0.2-3) per four weeks. Of 183 men who had ever performed ASE, 176 provided answers for ASE positions, and the most commonly reported position was standing $(50 \%, n=88)$, followed by squatting $(45 \%, n=80)$ (Fig. 1$)$. There were $28 \%(n=50)$ men who reported using more than one position for ASE, and the most common combination was standing and lying on their back $(5 \%, \mathrm{n}=8)$.

Most men performed ASE in the shower $(61 \%, n=106)$, followed by in the bathroom or toilet $(49 \%, n=85)$. Most men $(94 \%, n=163)$ reported using at least one accessory when performing ASE. The most commonly used accessory was lubricant including sorbolene and saliva $(59 \%, n=103)$, followed by water $(39 \%, n=68)$, soap $(26 \%, n=45)$, mirror $(24 \%, n=42)$, and gloves $(11 \%, n=19)$ during ASE (Table 2$)$. 
Table 2

Type of abnormalities felt during ASE, ease of performing the examination and likelihood of recommending it to other MSM.

\section{Experience related to ASE}

\section{Felt something during ASE}

Yes

No

\section{Description of abnormalities}

Lumps

Ulcers or sores

Bleeding

Others *

(Haemorrhoids $\times 7$, warts $\times 2$, tearx 1 , lump, varicose vein, tenderness)

\section{Ease of performing ASE}

Very easy

Easy

Neutral

Difficult

Very difficult

Likelihood of recommending ASE to other men by men who had ever performed ASE

Very likely

Likely

Maybe

Unlikely

Very unlikely
$\mathrm{N}=\quad$ Percentage

173

$81 \quad 47$

92

53

$N=79$

$50 \quad 63$

$22 \quad 28$

$30 \quad 38$

12

15

(\%)

7

53

12

$\mathrm{N}=$

172

$43 \quad 25$

$72 \quad 42$

$44 \quad 26$

$11 \quad 6$

$2 \quad 1$

$\mathrm{N}=$

167

(\%)

$74 \quad 44$

$60 \quad 36$

$21 \quad 13$

95

32

Of 173 men who had ever performed ASE, 47\% $(n=81)$ had ever felt something abnormal during ASE, with more than half of these men $(63 \%, n=50)$ reported the findings as a "lump", while $38 \%(n=30)$ reported bleeding and $28 \%(n=22)$ reported ulcers and/or sores (Table 2$)$. Most $(66 \%, n=115)$ found it 
easy or very easy to perform ASE. Only $8 \%(n=13)$ found it difficult or very difficult to perform ASEs (Table 2).

Men were presented with potential resources and information that might be useful for MSM who had never performed ASEs (e.g. graphics on poster or website showing how to do an examination, having a doctor or nurse speak in person and explain how to do ASE, or online videos from a trusted source (e.g. www.anal.org.au), see supplementary material). The top resource that men indicated would likely be helpful was an instructional graphics on posters or websites showing how to do ASE $(66 \%, n=113)$ (Table 3). Men were asked about information that might be useful to learn about anal ASE and syphilis such as how to do the examination, what a healthy anus should look, or what a syphilis lesion looks like (see supplementary material). Most men $(63 \%, \mathrm{n}=106)$ reported all the information related to ASE (e.g. how to perform, a healthy look of anus, and syphilis), anorectal syphilis (e.g. what a syphilis lesion looks like, what a syphilis lesion feels like), other non-STI symptoms of the anus and symptoms of anal cancer would be helpful. Table 3 showed the responses from the participants. 
Table 3

ASE positions, locations, resources, information among men, stratified by ever and never performing ASE

\begin{tabular}{|c|c|c|c|c|}
\hline \multirow[b]{2}{*}{ ASE positions } & \multicolumn{2}{|c|}{$\begin{array}{l}\text { Ever } \\
\text { performed* } \\
\text { ASE }(\mathrm{N}, \%)\end{array}$} & \multicolumn{2}{|c|}{ 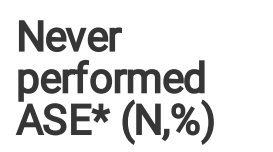 } \\
\hline & $N=176$ & $\%$ & $N=227$ & $\%$ \\
\hline Standing & 88 & 50 & 88 & 39 \\
\hline Squatting & 80 & 45 & 61 & 27 \\
\hline Lying on the side & 35 & 20 & 47 & 21 \\
\hline Lying on the back & 36 & 20 & 31 & 14 \\
\hline Others & 6 & 3 & $\mathrm{~N} / \mathrm{A}$ & \\
\hline Locations for performing ASE & $N=173$ & & $N=232$ & \\
\hline Shower & 106 & 61 & 151 & 65 \\
\hline Bed & 40 & 23 & 66 & 28 \\
\hline Bathroom/toilet & 85 & 49 & 105 & 45 \\
\hline Others & 4 & 2 & 3 & 1 \\
\hline Resources relating to ASE technique ${ }^{\wedge}$ & $N=171$ & & $N=328$ & \\
\hline Instructional graphics on poster or website & 113 & 66 & 222 & 68 \\
\hline In-person explanation from doctor/nurse & 82 & 48 & 172 & 52 \\
\hline $\begin{array}{l}\text { In-person demonstration by a doctor/nurse using a training } \\
\text { model/manikin }\end{array}$ & 31 & 18 & 85 & 26 \\
\hline Videos of a doctor/nurse using a training model or a live person & 64 & 37 & 162 & 49 \\
\hline $\begin{array}{l}\text { Online videos from a trusted source (i.e. existing and available to } \\
\text { general public) (e.g., www.anal.org.au) }\end{array}$ & 81 & 47 & 182 & 55 \\
\hline Explanation from partners or friends & 23 & 13 & 17 & 5 \\
\hline Do not need any resources & 4 & 2 & 13 & 4 \\
\hline Information relating to ASE and anal syphilis & $N=168$ & & $N=325$ & \\
\hline How to do the examination & 44 & 26 & 105 & 32 \\
\hline \multicolumn{5}{|l|}{ \% Percentage } \\
\hline \multicolumn{5}{|c|}{$\begin{array}{l}\text { *The two groups were not comparable as they were presented with different scenarios such as } \\
\text { positions already used by men who had performed ASE vs positions likely to be used by men who } \\
\text { had never performed ASE. }\end{array}$} \\
\hline
\end{tabular}




\begin{tabular}{|lllll|}
\hline & $\begin{array}{l}\text { Ever } \\
\text { performed* } \\
\text { ASE (N,\%) }\end{array}$ & $\begin{array}{l}\text { Never } \\
\text { performed } \\
\text { ASE* (N,\%) }\end{array}$ \\
\hline Where to do the examination & 11 & 7 & 21 & 6 \\
\hline What a healthy anus should look like & 43 & 26 & 106 & 33 \\
\hline What a syphilis lesion looks like & 42 & 25 & 101 & 31 \\
\hline What a syphilis lesion feels like & 42 & 25 & 105 & 32 \\
\hline $\begin{array}{l}\text { What other sexually transmitted infections (STI) symptoms would } \\
\text { look or feel like on the anus, including warts and herpes }\end{array}$ & 0 & 0 & 80 & 25 \\
\hline Other non-STI symptoms of the anus (e.g., haemorrhoids) & 31 & 18 & 75 & 23 \\
\hline Symptoms of anal cancer & 32 & 19 & 80 & 25 \\
\hline All of the above & 106 & 63 & 193 & 59 \\
\hline \% Percentage & & & & \\
\hline $\begin{array}{l}* \text { The two groups were not comparable as they were presented with different scenarios such as } \\
\text { positions already used by men who had performed ASE vs positions likely to be used by men who } \\
\text { had never performed ASE. }\end{array}$ & & & \\
\hline ^ allowed multiple options & & & \\
\hline
\end{tabular}

Among men who had ever performed ASE, 167 men answered the question about recommending ASE to other MSM, and $80 \%(n=134)$ indicated that they were likely or very likely to recommend other MSM to perform ASE.

\section{Preferences that men who had never performed ASE likely to have if they were to perform ASE}

Men who had never performed ASE were asked if they would consider ASEs regularly at the recommendation of a doctor to detect syphilis sores or ulcers. Among the $\mathbf{3 7 4}$ men who never performed anal self-examinations, $68 \%$ (250) would consider anal self-examinations in the future for anal syphilis detection, while $18 \%$ (65) would like more information about the examination. Nine percent (35) were unable to decide if they would consider anal self-examination and $5 \%(20)$ did not want to perform anal self-examinations at all.

Of the 374 men who had never performed ASE, 28\% $(n=105)$ men engaged in receptive anal sex; $52 \%$ ( $n$ $=193$ ) men engaged in versatile anal sex. Among 298 men engaging in receptive or versatile anal sex and never performed ASE, 69\% $(n=205)$ would consider performing ASE to detect early syphilis in the future, while $18 \%(n=54)$ would like to receive more information about ASE before making the decision. Eight percent $(n=24)$ were unable to decide if they would consider performing ASE, and $4 \%(n=12)$ did not want to perform ASE at all. 
Among the men recruited from the community and never performed ASE $(n=36), 44 \%(n=16)$ indicated they would be willing to perform ASE, while $52 \%(n=19)$ wanted more information or unsure about performing ASE in future (data not shown in results) and $3 \%(n=1)$ did not want to perform ASE in future.

Of the 250 men who were willing to consider performing ASE in the future regardless of their sex position, the median of the preferred frequency for ASE was two times per four weeks (IQR: 1-4). Among these men, 205 men engaged in either receptive or versatile anal sex, and the median of the preferred frequency for ASE was four times per four weeks (IQR: 1-4).

There were 227 men who had never performed ASE indicated they would most likely prefer standing $(39 \%, n=88)$ if they were to perform ASE in the future, followed by squatting $(27 \%, n=61)$ (Fig. 1).

Of the 232 men who answered the questions on locations they most likely to perform ASE, $65 \%(n=151)$ reported the shower as the preferred location where they were likely to perform ASE, followed by bathroom or toilet $(45 \%, n=105)$ (Table 3). Almost half $(43 \%, n=98)$ would consider having their partner (regular romantic or regular sex partners) examine their anus when men were asked if they would let their partner examine their anus. Only a few $(8 \%, n=17)$ would consider a casual partner performing the examination.

When presented with choices of who they prefer to be performing an anal examination for an abnormality, 45\% ( $n=53)$ of the 117 men who answered the question preferred to have a doctor performed an anal examination to check for syphilis lesions in their anus, although some men $(28 \%, \mathrm{n}=$ 33) preferred performing anal examination themselves and $27 \%(n=31)$ did not have a preference.

Most men $(68 \%, \mathrm{n}=222)$ out of 328 men answering questions about information about ASE and resources, would like at least some information about ASE (how to perform ASE, locations to perform ASE, and how to distinguish between normal and abnormal findings) and anorectal syphilis with $59 \%$ ( $\mathrm{n}$ $=193$ ) wanting all the information about ASE, syphilis lesions, other non-STI symptoms of anus and symptoms of anal cancer (Table 2). The majority $(96 \%, n=315)$ of men who had never performed ASE would like to learn from various resources such as through a website with instructional graphics, online videos, through doctors/nurses, and a small number reported willing to learn from friends or partners, whilst only $4 \%(n=4)$ reported not needing any resources (Table 3$)$.

Of the 324 men who had never performed ASE, the majority were very likely or likely to consider performing ASE in the future $(89 \%, n=288)$, whilst $2 \%(n=6)$ chose not to perform ASE in the future, and $9 \%(n=20)$ were unable to decide.

\section{Discussion}

Our study shows that about two-thirds of MSM (65\%) who participated in the survey had never performed ASE, although the majority (88\%) of these men were interested in performing ASE in the future for detecting anal syphilis lesions and most preferred to do it once per month. Men who had ever performed 
ASE largely found it easy to perform ASE, and most would recommend performing ASE to other MSM. Although various ASE positions were reported, the two groups of men who had ever performed and who had never performed ASE uniformly preferred the standing position, followed by the squatting position. Men in both groups preferred performing ASE in the shower compared to other locations. Most men would like to learn or gather more information about how to perform ASE on more accessible forms of learning such as videos, graphics on a poster, and a website instead of in-person training such as a doctor or nurse demonstrating face-to-face training. Men also expressed wanting to get more information about syphilis, especially anal syphilis and the abnormalities that likely to encounter during ASE. There was no difference between the two groups in terms of age, HIV status and PrEP use, implying that future public health campaigns should target everyone rather than a specific population.

The positive attitudes towards ASE for syphilis detection reported here are consistent with our previous qualitative study of MSM on ASE ${ }^{21}$. Similarly, the study by Ong et al. showed a high level of acceptance towards performing ASE for anal cancer screening ${ }^{20}$. The ongoing syphilis epidemic among MSM, highlight the need for novel strategies for the early detection of syphilis that will reduce the duration of infectiousness ${ }^{22}$.

Before proceeding to implement ASE, it would be first important to establish that men could detect primary syphilis lesions in the anal canal. If shown to be effective, ASE for syphilis detection would be a new concept and intervention for MSM; educating MSM on performing ASE correctly would be required. Most men preferred this information to be publicly available on a poster or website rather than gathering this information from health professionals.

Before implementing such a program, it would also be important to determine how commonly men presenting with findings that were not primary syphilis or other conditions of concern. This would be an important consideration because it would influence the cost and cost-effectiveness of any such program. If this was very common, then it may limit the usefulness of such a program.

There are several limitations to the study. First, most of the participants were recruited from a sexual health clinic, and only a small proportion was recruited from the community. Therefore, we might not be able to generalise the finding to the whole MSM community, as men attending a sexual health centre may be more health-conscious and perhaps more willing to perform ASE. Among the men recruited from the community and never performed ASE, almost half (44\%) indicated willingness to perform ASE compared to $68 \%$ of sexual health centre attendees. Over half $(52 \%)$ of the men recruited from the community wanted more information or were unsure about ASE implying that more health promotion and education about ASE might be required if ASE were to be recommended in the future. Second, the survey was limited to participants from Australia to minimise spam responses from outside of Australia, and therefore the findings can only apply to MSM residing in Australia. Third, we did not know why $4 \%$ of men reported that they would not consider performing ASE in the future, as this information was not collected. Finally, the survey had a low participation rate which may have biased the responses, particularly for example towards the percentage of men who had previously performed ASE. 


\section{Conclusion}

This study found that most men who had never performed ASE are willing to perform ASE to detect any abnormalities in the future. If ASE is shown to be effective and cost-effective in detecting early anal syphilis lesions, this could be translated into a public health intervention entailing ASE combined with regular STI screening.

\section{Method}

\section{Study population and distribution}

We conducted a cross-sectional anonymous online survey named "Self-Examination of Anal Syphilis - a Survey study" (SEAS-S) between July and November 2020. Men who self-reported having sex with other men, aged 18 and above, and living in Australia were eligible to participate in the survey. Men were recruited from the Melbourne Sexual Health Centre (MHSC), Victoria, Australia during the study period. All men attending MSHC were asked whether they would like to receive additional information (e.g., research studies) via SMS with their registered mobile number. An SMS explaining the SEAS-S study and a link to the online survey was sent to all men who provided consent to receive additional information each day during the study period. Furthermore, men were also recruited from the general population across Australia. A link to the survey was posted and distributed through social media (e.g., Facebook, Twitter) as well as internal social networks with community-based organisations supporting lesbian, gay, bisexual, transgender, intersex, queer people or questioning (LGBTIQ+) communities and people living with HIV in Australia.

Ethical approval was obtained from the Alfred Hospital Ethics Committee, Melbourne, Australia (366/20) and endorsement obtained from Thorne Harbour Health, a community organisation in Victoria, Australia (THH/CREP 20-013). All research was performed in accordance with the relevant guidelines/regulations of the Alfred Hospital Ethic Committee.

Informed consent was obtained from all the participants (see under survey information outlining the process of obtaining informed consent).

\section{Survey information}

The first page of the online survey described the detailed nature of the study, including a participant information sheet that the participants could download. Participants were required to provide consent to participate in the survey by selecting the 'Agree' button on the first page before commencing the survey. Participants who decided not to participate could select the 'Disagree' button to terminate the survey.

The survey collected demographic characteristics such as age, country of residence, gender, sexual orientation, HIV status and currently taking pre-exposure prophylaxis (PrEP) for HIV, and whether the participants had ever performed ASE. We defined ASE as individuals inserting a finger into their anus to 
check for abnormalities. This was an anonymous survey, and no other personally identifiable information was collected in the survey.

In our qualitative study which is part of the series of anal examination study for detection of anorectal syphilis ${ }^{21}$, men who reported inserting their fingers for pleasuring themselves noticed abnormal findings during the examination. Therefore, in this study, we only asked about ASE performed for the purpose of detecting abnormalities. Those who had ever performed ASE were asked about the positions, accessories, and locations when they performed ASE, and resources and information likely to be helpful for men who were new to ASE. Participants who had never performed ASE were asked about the possible positions and locations that they would likely use to perform ASE and resources and information that they might find useful to learn ASE.

For analysis purpose, we included surveys with minimum completion of some demographics such as gender, sexual orientation, sex position, education, and HIV and PrEP status, whilst the surveys that did not meet the minimum completion were excluded.

The survey was generated and conducted using the Qualtrics platform (Qualtrics, Provo, UT), which collected the location latitude and longitude automatically where the participant completed the survey. The location latitude and longitude were converted to the location of the city. Participants with a location outside Australia were excluded from this analysis.

\section{Statistical analysis}

Descriptive statistics were computed to summarise the demographic characteristics and study variables. Men were categorised into two groups based on whether they had ever performed ASE: 'ever performed' and 'never performed'. Demographic characteristics between men who had ever performed and those who had never performed ASE were compared using Fisher's exact test for categorical variables and Mann-Whitney $U$ test for continuous variables. Individuals were able to decline to answer any questions in the survey and they were considered as missing data. The proportion of the study variables was calculated based on the number of participants who answered that question excluding those who declined to answer. All analyses were conducted with STATA (StataCorp. 2019. Stata Statistical Software: Release 16. College Station, TX: StataCorp LLC.)

\section{Declarations}

\section{Data availability}

All data generated or analysed during this study are included in this published article (and its Supplementary Information files).

\section{Acknowledgement}


We would like to thank Mark Chung for his expertise in designing graphics for the survey and promotion material, Thorne Harbour Health for assistance with promotion study in the community through social media.

\section{Author contribution}

CKF, EPFC, TPP, JJO, and ETA designed and coordinated the study. ETA developed the survey and drafted the manuscript. ETA, KM and ERR distributed the survey. ETA and EPFC performed the analysis of the data and revised the manuscript. All authors read and approved the final manuscript.

\section{Competing Interests Statement}

This study was supported by an Australian National Health and Medical Research Council (NHMRC) Investigator Grant (GNT1172873).

- Financial interests: All other authors have no financial conflicts of interest to declare.

- Non-financial interest: EPFC is supported by an Australian NHMRC Emerging Leadership Investigator Grant (GNT1172873). CKF is supported by an Australian NHMRC Leadership Investigator Grant (GNT1172900). JJO is supported by an Australian NHMRC Early Career Fellowship (GNT1104781). ETA is supported by Australian Government Research Training Program (RTP) scholarship from Monash University and Research Entry Scholarship from the Chapter of Sexual Health Medicine, Royal Australasian College of Physician. JT is supported by Australian Government Research Training Program (RTP) Scholarship. All other authors have no conflicts of interest to declare.

\section{References}

1. Kirby Institute. HIV, viral hepatitis and sexually transmissible infections in Australia Annual surveillance report 2018 (Kirby Institute, UNSW, Sydney, 2018a).

2. Aung, E. T. et al. Spatial and temporal epidemiology of infectious syphilis in Victoria, Australia, 2015-2018. Sexually Transmitted Diseases Publish Ahead of Print, doi:10.1097/olq.0000000000001438 (9000).

3. Kojima, N. \& Klausner, J. D. An Update on the Global Epidemiology of Syphilis. Curr Epidemiol Rep, 5, 24-38 https://doi.org/10.1007/s40471-018-0138-z (2018).

4. Williamson, D. A. \& Chen, M. Y. Emerging and Reemerging Sexually Transmitted Infections. New England Journal of Medicine, 382, 2023-2032 https://doi.org/10.1056/NEJMra1907194 (2020).

5. Chow, E. P. F. P., Grulich, A. E. P. \& Fairley, C. K. P. Epidemiology and prevention of sexually transmitted infections in men who have sex with men at risk of HIV. Lancet HIV, The, 6, e396-e405 https://doi.org/10.1016/S2352-3018(19)30043-8 (2019).

6. Bissessor, M., Fairley, C. K., Leslie, D., Howley, K. \& Chen, M. Y. Frequent screening for syphilis as part of HIV monitoring increases the detection of early asymptomatic syphilis among HIV-positive 
homosexual men. Journal of acquired immune deficiency syndromes (1999), 55, 211-216 https://doi.org/10.1097/qai.0b013e3181e583bf (2010).

7. Down, I. et al. Increasing gay men's testing rates and enhancing partner notification can reduce the incidence of syphilis. Sex Health, 9, 472-480 https://doi.org/10.1071/sh12023 (2012).

8. McCann, P. D. et al. Would gay men change their sexual behavior to reduce syphilis rates? Sex. Transm. Dis, 38, 1145-1150 https://doi.org/10.1097/OLQ.0b013e318238b846 (2011).

9. Gunn, R. A. \& Klausner, J. D. Enhancing the Control of Syphilis Among Men Who Have Sex With Men by Focusing on Acute Infectious Primary Syphilis and Core Transmission Groups. Sex Transm Dis, 46, 629-636 https://doi.org/10.1097/OLQ.0000000000001039 (2019).

10. Cox, D. \& Ballard, R. C. in Atlas of Sexually Transmitted Diseases and AIDS (Fourth Edition) (eds Stephen A. Morse, Ronald C. Ballard, King K. Holmes, \& Adele A. Moreland)111-140(W.B. Saunders, 2010).

11. Cornelisse, V. J. et al. Getting to the Bottom of It: Sexual Positioning and Stage of Syphilis at Diagnosis, and Implications for Syphilis Screening. Clinical infectious diseases: an official publication of the Infectious Diseases Society of America, 71, 318-322 https://doi.org/10.1093/cid/ciz802 (2020).

12. Gunn, R. A. \& Peterman, L. M. TA, in 2006 National STD Prevention Conference (Jacksonville, FL: May 8-11 2006).

13. Peel, J. et al. Clinical presentation of incident syphilis among men who have sex with men taking HIV Pre-Exposure Prophylaxis in Melbourne, Australia. Clinical infectious diseases: an official publication of the Infectious Diseases Society of America, https://doi.org/10.1093/cid/ciab052 (2021).

14. Towns, J. M. et al. Treponema pallidum detection in lesion and non-lesion sites in men who have sex with men with early syphilis: a prospective, cross-sectional study. Lancet Infect Dis, https://doi.org/10.1016/s1473-3099(20)30838-0 (2021).

15. Ong, J. J. et al. Cost-effectiveness of screening for anal cancer using regular digital ano-rectal examinations in men who have sex with men living with HIV. J Int AIDS Soc, 19, 20514 https://doi.org/10.7448/IAS.19.1.20514 (2016).

16. Ong, J. J. et al. Baseline findings from the Anal Cancer Examination (ACE) study: screening using digital ano-rectal examination in HIV-positive men who have sex with men. J Med Screen, 23, 70-76 https://doi.org/10.1177/0969141315604658 (2016).

17. Ong, J. J. et al. Incorporating digital anorectal examinations for anal cancer screening into routine HIV care for men who have sex with men living with HIV: a prospective cohort study. J Int AIDS Soc, 21, e25192 https://doi.org/10.1002/jia2.25192 (2018).

18. Nyitray, A. G., D'Souza, G., Stier, E. A., Clifford, G. \& Chiao, E. Y. The Utility of Digital Anal Rectal Examinations in a Public Health Screening Program for Anal Cancer. Journal of Lower Genital Tract Disease, 24, 192-196 https://doi.org/10.1097/lgt.0000000000000508 (2020).

19. Nyitray, A. G. et al. A phase II clinical study to assess the feasibility of self and partner anal examinations to detect anal canal abnormalities including anal cancer. Sex Transm Infect, 94, 124- 
130 https://doi.org/10.1136/sextrans-2017-053283 (2018).

20. Ong, J. J. et al. Exploring anal self-examination as a means of screening for anal cancer in HIV positive men who have sex with men: a qualitative study. BMC Public Health, 14, 1257 https://doi.org/10.1186/1471-2458-14-1257 (2014).

21. Aung, E. et al. in Poster presented at HI\&AIDS and Sexual Health Conferences; 2020 November 1620; Virtual, Australia(2020).

22. Chow, E. P. F., Grulich, A. E. \& Fairley, C. K. Epidemiology and prevention of sexually transmitted infections in men who have sex with men at risk of HIV. Lancet HIV, 6, e396-e405 https://doi.org/10.1016/s2352-3018(19)30043-8 (2019).

\section{Figures}

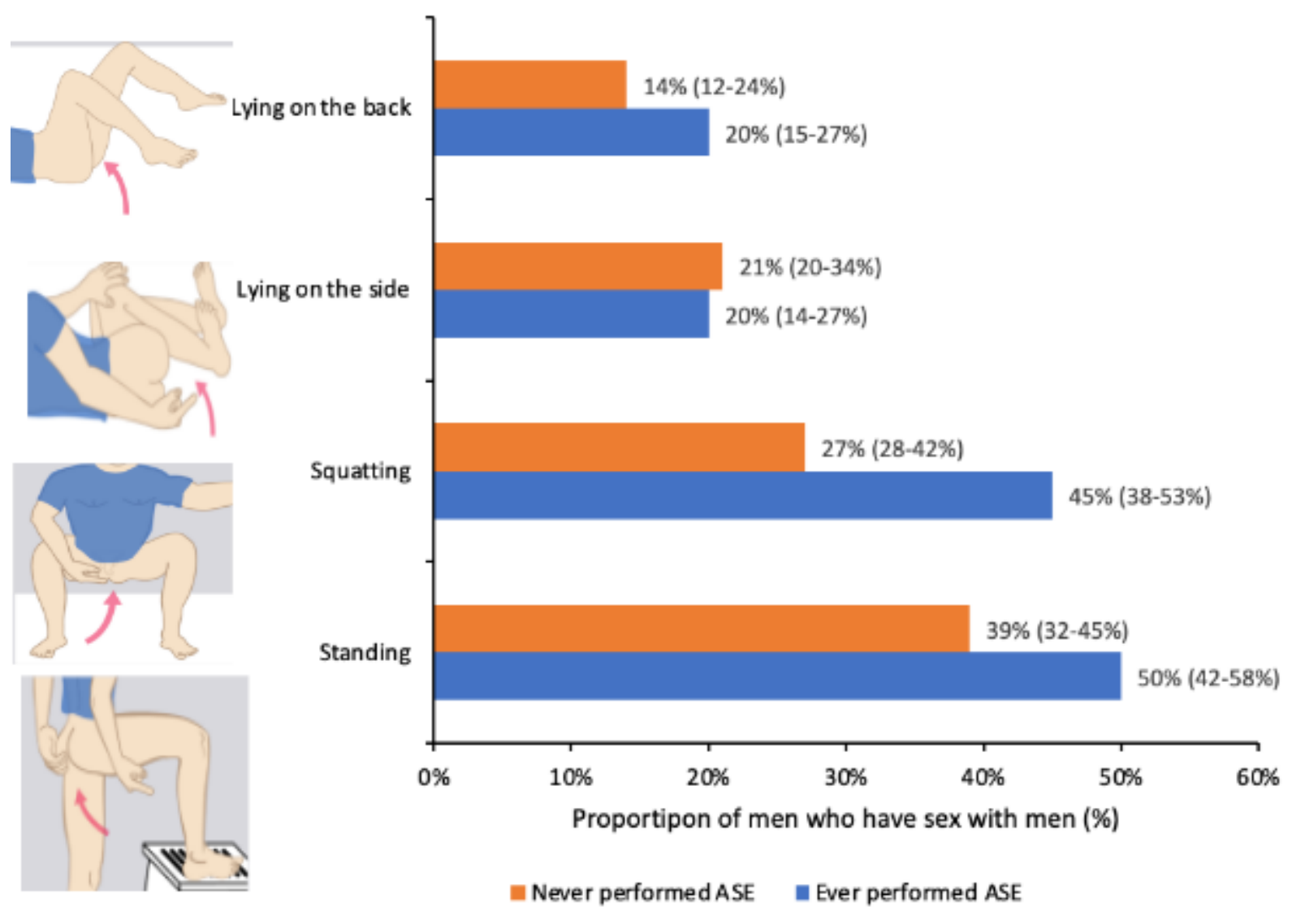

Figure 1

positions used by MSM who had ever performed ASE and positions likely to be used by MSM who had never performed ASEs 95\% confidence interval expressed in brackets following the proportion. Note: 176 men who had ever performed ASE and 227 who had never performed ASE answered questions on sexual positions. Six men in the ever-performed ASE chose other positions. Other positions were not asked in the never performed ASE group. This category was not included in the graph. 


\section{Supplementary Files}

This is a list of supplementary files associated with this preprint. Click to download.

- Scireportsupplementary.pdf 